\title{
The Lattice Boltzmann Method used for fluid flow modeling in hydraulic components
}

\author{
Bernhard Manhartsgruber \\ Institute of Machine Design and Hydraulic Drives \\ Johannes Kepler University, Linz, Austria \\ E-mail: bernhard.manhartsgruber@jku.at
}

\begin{abstract}
The Lattice-Boltzmann Method for the approximate solution of the Navier-Stokes equations has become an interesting alternative to classical finite volume based discretization methods. Because the flow domain is not meshed in the classical sense but only voxelized and geometrically complex boundaries can be introduced in an easy form by bounce-back or offLattice boundary conditions, the method lends itself very well to simulations of channel flows inside hydraulic components. In this paper, a flow problem in a single acting cylinder attached to a $3 / 2$ directional spool valve is used as a benchmark problem. The Lattice-Boltzmann simulation is used to generate a reference solution for the pressure step response of the blocked cylinder with superimposed wave propagation. From this reference data set, a non-parametric frequency domain input-output model is extracted and compared with results from classical lumped parameter modeling.
\end{abstract}

Keywords: Lattice-Boltzmann method, fluid power systems modeling

\section{Introduction}

The Lattice-Boltzmann method evolved out of the early ideas of Lattice gas automata [1, 2, 3] from the 1970s and early 1980s. These models were discrete in the sense that they took into account only binary information about presence or absence of a particle at a grid point in a certain time step. The very fine lattice resolution needed to produce a solution with acceptable molecular noise rendered the method inapplicable to engineering problems. However, the combination of the lattice-gas idea with a description of the fluid by the Boltzmann equation [4] resulted in a very powerful tool over the last two decades. The central idea is the description of the state of the fluid by probability densities with a simple stream and collide mechanism for updating the information from one time level to the next.

Due to the local nature of the description and the absence of the solution of large equations systems, the Lattice Boltzmann method (LBM) is very well suited for implementation on parallel computing systems. The present paper shows the use of LBM solutions of a channel flow problem including wave propagation effects for the generation of low order state space models by identification and order reduction methods. This novel approach includes minor pressure losses and wave propagation effects that are difficult to handle in classical lumped parameter modelling. Yet, the resulting mathematical models are of very low order as compared to CFD modeling.

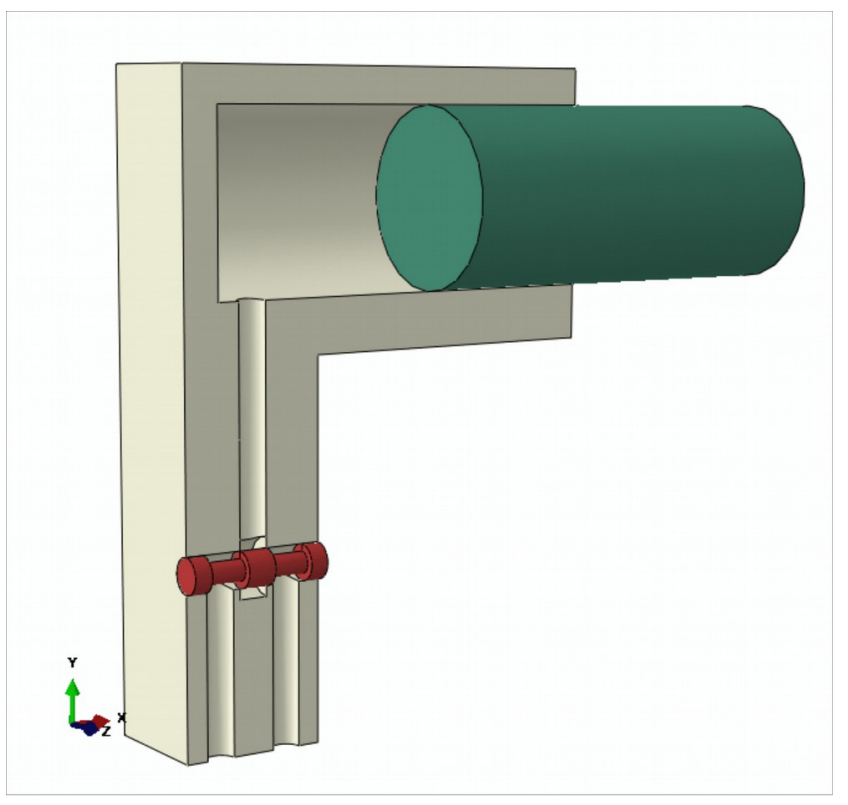

Figure 1: Geometry of the example problem. 


\section{The example problem}

Fig. 1 shows the geometry of the example problem. A 3/2 directional spool valve controls the pressure in a cylinder chamber. The supply pressure and tank pressure attached to the valve ports at the bottom of the geometry are assumed constant. The chamber pressure controlled by the spool valve acts against an external force pushing the piston inwards.

The compuational domain shown in Fig. 2 consists of four sections:

- $\quad$ an $8 \mathrm{~mm}$ supply bore between a constant pressure boundary condition and the spool valve with a length of $45 \mathrm{~mm}$

- the spool valve geometry, consisting of two annular chambers (diameters $6 \mathrm{~mm} / 10 \mathrm{~mm}$ and $10 \mathrm{~mm} 16 \mathrm{~mm}$ ) with an axial valve opening of 1 $\mathrm{mm}$

- $\quad$ an $8 \mathrm{~mm}$ connection bore between the spool valve and the piston chamber with a length of $62 \mathrm{~mm}$

- the piston chamber with a cylindrical geometry of diameter $50 \mathrm{~mm}$ and height $30 \mathrm{~mm}$ (the height is acutally varialbe due to piston movement. Figures 1 and 2 show a longer piston chamber, the compuations results presented in this paper are for only $30 \mathrm{~mm}$ of height.

The goal pursued in this paper is to describe the pressure build-up in the cylinder chamber due to the valve opening in a precise way including minor losses at the connection of the bore between valve and cylinder chamber. Furthermore, the high frequency dynamics of wave propagation between the valve and the piston face should be included as far as possible in a low order model.

The approach followed to reach this goal is as follows:

- In a first step, a number of different piston motions due to valve opening have to be simulated using the Lattice-Boltzmann method. In the present paper only a first result for the blocked piston case is presented.

- From the simulation results, effective piston pressure and valve flow signals are computed.

- These signals are used to identify low order state space models describing the input-ouput behaviour of the hydraulic system.

The resulting models are in the end compared to lumped parameter modelling using just a pressure build-up equation for the cylinder chamber as described in classical fluid power text books, see e. g. [5].

\section{Simulation using LBM}

This paper uses a simplified approach as the motion of the valve spool and hydraulic piston is disregarded. The simulated case corresponds to the step response of the cylinder chamber pressure with blocked piston motion after an instantaneous opening of the valve. The boundary of the flow domain shown in Fig. 2 is constant in time and can be described in a very simple way as surface triangulation data in a stereo lithography (.stl) file provided by many CAD systems.

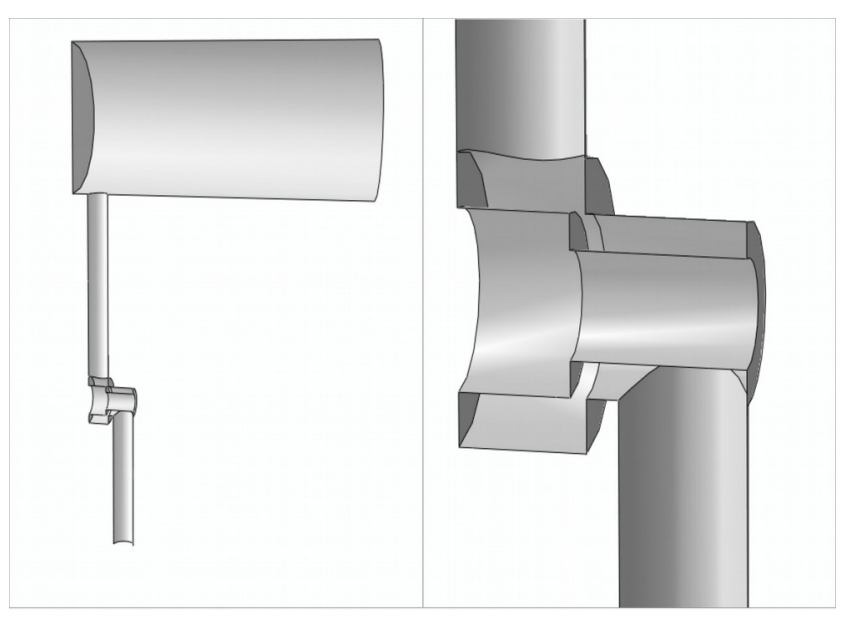

Figure 2: Geometry of the bounding walls in .stl file.

The left half of Fig. 2 shows the overall boundary geometry of the flow domain with two open faces, one at the bottom, where the bore supplying pressurized hydraulic fluid to the valve ends, and one at the top right corner, where the fluid faces the piston. The first open face is treated as a constant pressure boundary condition, while the second one is understood as a velocity boundary, where the fluid velocity normal to the boundary patch can be prescribed as a flow profile. For the case presented here with the piston motion blocked - the flow profile will be prescribed as zero over the whole patch which is identical to using a wall boundary condition as for all the other parts of the boundary shown as shaded patches in Fig. 2. the reason for specifying the boundary with two open patches and both a pressure and a velocity boundary patch lies in the use of the Lattice-Boltzmann code PALABOS. The computation presented here uses a showcase of this software package where the flow through an aneurysm geometry is analysed [6]. This showcase readily implements the geometry import from the stereolithography (.stl) file and needs at least one inflow patch with prescribed velocity and one outlet with prescribed pressure. This setting is inverted in the present case in the sense that the inflow is blocked by setting the velocity profile to zero and the pressure at the former outflow patch may result in a velocity pointing into the flow domain reverting this boundary patch to an inflow port.

With this approach, the "aneurysm" showcase of the PALABOS software package, which can be found in the examples folder of every standard installation, is used as a starting point. By exchanging the geometry file of the 
"aneurysm" showcase with the data shown in Fig. 2 and adding a few lines of code for writing the average pressure at the piston face and the average pressure and flow rate at the control edge of the valve (see the zoomed picture in the right hand side of Fig. 2) to a file at a certain sample rate during the simulation.

Lattice-Boltzmann calculations are carried out in a dimensionless framework where the time evolves in unit steps by a so called collide and stream procedure and the space is divided into cubic voxels of edge length one. The scaling between this voxelization of the flow domain and the physical length scales is represented by the spatial resolution $d x$. The dimensionless speed of sound in the lattice has a fixed value of

$$
c_{L B}=\frac{1}{\sqrt{3}} .
$$

In order to match this lattice speed of sound with the physical speed of sound, the grid viscosity is set to

$$
v_{L B}=\frac{v}{\sqrt{3 \frac{K}{\rho} \cdot d x}}
$$

The physical time step follows as

$$
d t=\frac{v_{L B}}{v} \cdot d x^{2}
$$

The value of the grid viscosity influences the stability of a Lattice-Boltzmann simulation. Numerical experiments with the PALABOS code used for the present paper showed that the calculations went unstable for values below $v_{\mathrm{LB}}=0.006$. Whether this limit could be lowered by using a different implementation fo the Lattice-Boltzmann method has not been reserached in the scope of this paper. The limit for the grid viscosity poses a severe problem with respect to the necessary spatial resolution for the calculations: The speed of sound must be matched in order to resolve the wave propagation in the right way. Setting $v_{\mathrm{LB}}$ to 0.006 results in

$$
d x=\frac{v}{0.006} \cdot \sqrt{\frac{\rho}{3 K}}
$$

With a typical kinematic viscosity of a mineral oil based hydraulic fluid of $v=40 \cdot 10^{-6} \mathrm{~m}^{2} / \mathrm{s}$, a mass density of $\rho=$ $850 \mathrm{~kg} / \mathrm{m}^{3}$, and an effective bulk modulus of $\mathrm{K}=1.5 \cdot 10^{9}$ $\mathrm{Pa}$, the lower limit for the grid viscosity around $v_{\mathrm{LB}}=0.006$ calls for a spatial resolution of $\mathrm{dx}=2.9 \cdot 10^{-6} \mathrm{~m}$. Together with the physical extent of the computational domain shown in Fig. 2 of 170 by 50 by $30 \mathrm{~mm}$, this results in a size of approximately 59000 by 17000 by 10000 grid cells which is way beyond the reach of available computing power.

In a first attempt to validate the tool chain of LatticeBoltzmann simulation and frequency domain identification, the kinematic viscosity of the fluid is vastly increased to a value of $1725 \cdot 10^{-6} \mathrm{~m}^{2} / \mathrm{s}$ resulting in a spatial resolution of $0.125 \mathrm{~mm}$ which gives a computational domain of 1360 by 400 by 240 cells in the overall lattice.

Clearly, this approach will not work for the simulation of fluid power systems in a realistic parameter range. The research towards implementations of Lattice-Boltzmann methods capable of dealing with a lower value of the grid viscosity while maintaining numerical stability is hower left to future work.

In order to give the reader a first impression of the method, Fig. 3 shows five sections through the computational domain at time steps $0,24,48,72$, and 96 respectively. The physical time step can be evaluated as

$$
d t=\frac{0.006}{1725 \cdot 10^{-6} \frac{\mathrm{m}^{2}}{\mathrm{~s}}} \cdot(0.00125 \mathrm{~m})^{2}=5.435 \cdot 10^{-8} \mathrm{~s}
$$

so the 96 time steps that have gone by in the rightmost part of Fig. 3 correspond to 5.2 microseconds. The colour code shows the region initially at high pressure in red and the low pressure region in blue. The initiation of a pressure wave propagating from the opened valve in both directions is clearly visible.

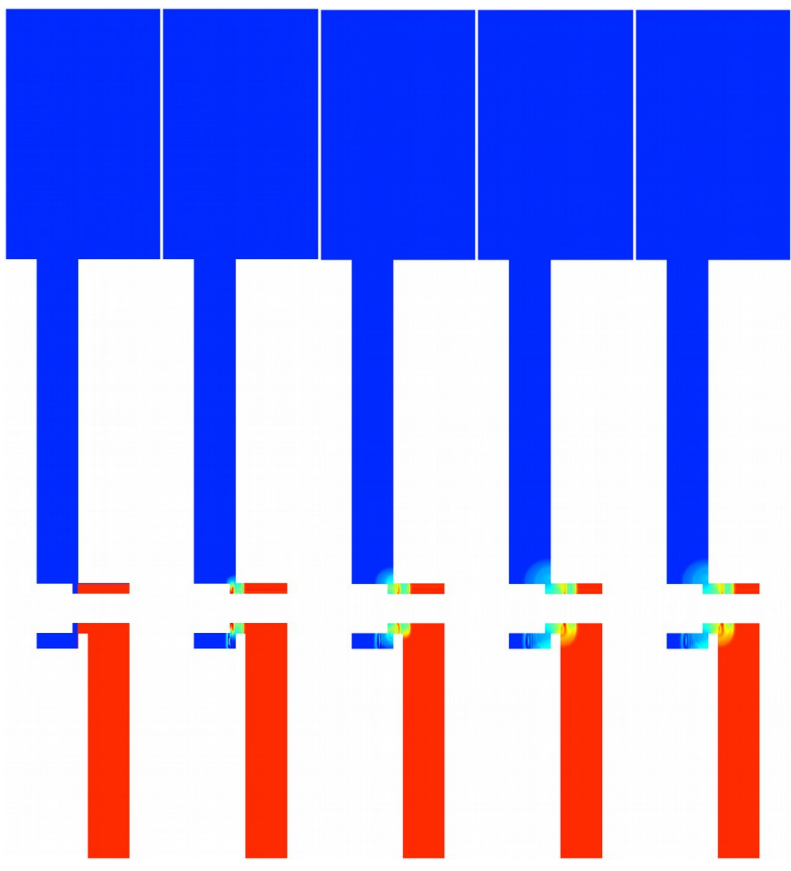

Figure 3: Pressure wave propagation shortly after initial condition (0, 24, 48, 72, 96 time steps).

Figure 4 shows the same colour coded pressure information but on a longer time scale up to 1500 time steps corresponding to roughly 82 microseconds in real time. The pressure waves travel into the two bores and are seen to be reflected at the bottom boundary condition as well as at the entry to the piston chamber which constitutes more ore less a constant pressure boundary condition on these small time scales. The velocity magnitude information is given for the same time steps in Fig. 5. 
The simulation must then be run for a very large number of time steps, as the material presented so far only resembles the first moment in a flow process supplying fluid to the cylinder chamber via the control edge of the valve from the constant pressure boundary condition at the bottom.

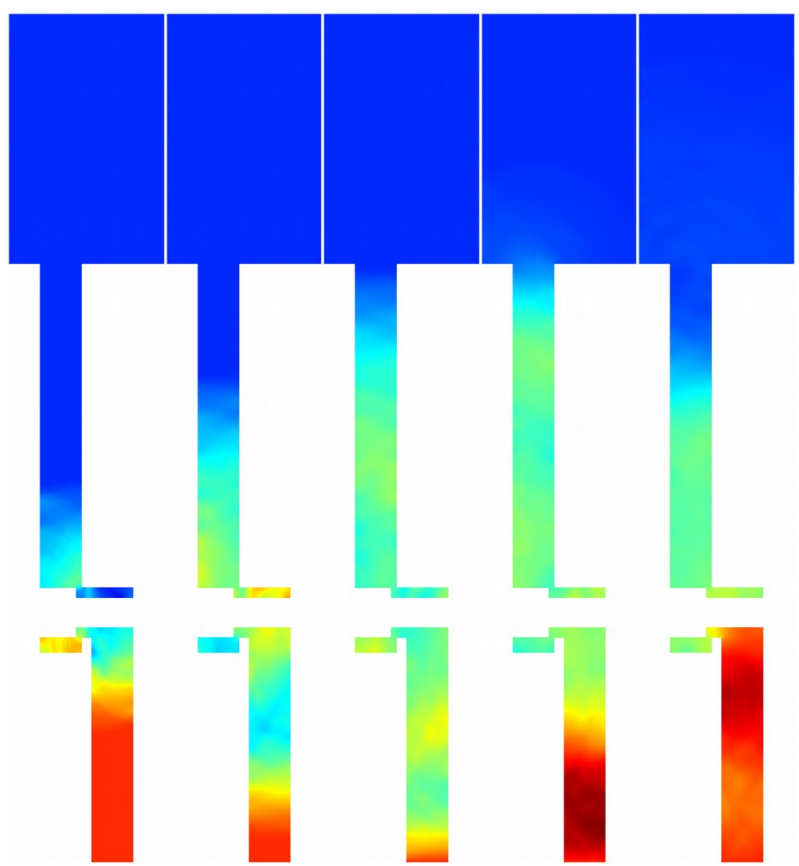

Figure 4: Pressure wave propagation at 300, 600, 900, 1200, 1500 time steps after initial condition.
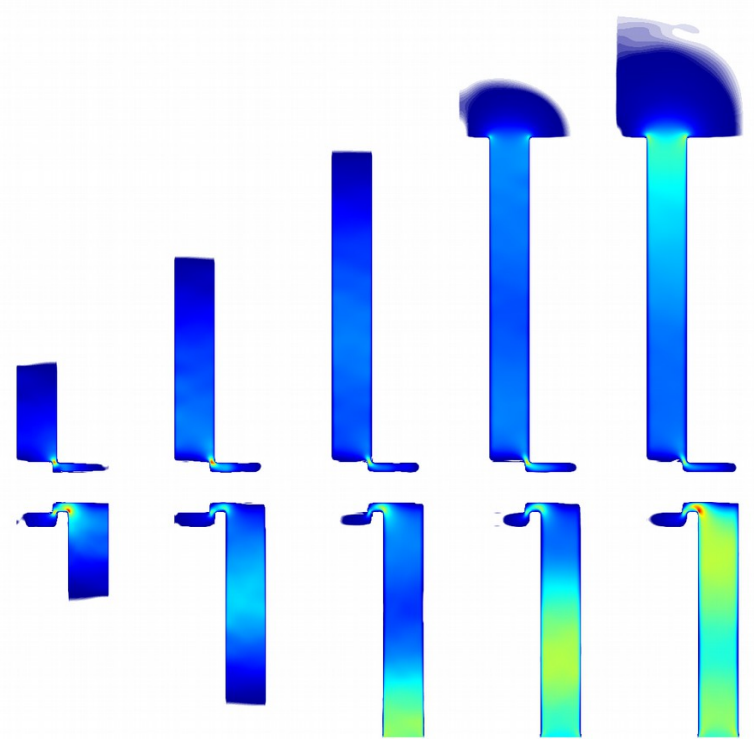

Figure 5: Magnitude of the flow velocity at 300, 600, 900, 1200, 1500 time steps after initial condition.

In order to condense the information into a low order model, the first step is the limitation of observations to input and output ports. The subsystem between the control edge of the valve and the piston face is cut out by first defining port patches and then measuring average pressure values at the patches and the net volumetric flow rates through them.

The port patch at the piston end is easily defined as the circular cross sectional area where the fluid touches the piston. In the case of the port patch defined inside the valve geometry, the geometric definition becomes clear from the initial condition of the pressure shown in the leftmost part of Fig. 3. The port patch is an annular area between the initial high and low pressure. The average pressure values and net flow rates through the two ports are given in Fig. 6 in practical engineering units of bar and 1/min. The index "In" in Fig. 6 corresponds to the input at the valve orifice while "Out" corresponds to the piston face. Clearly the flow rate at the blocked piston face vanishes.
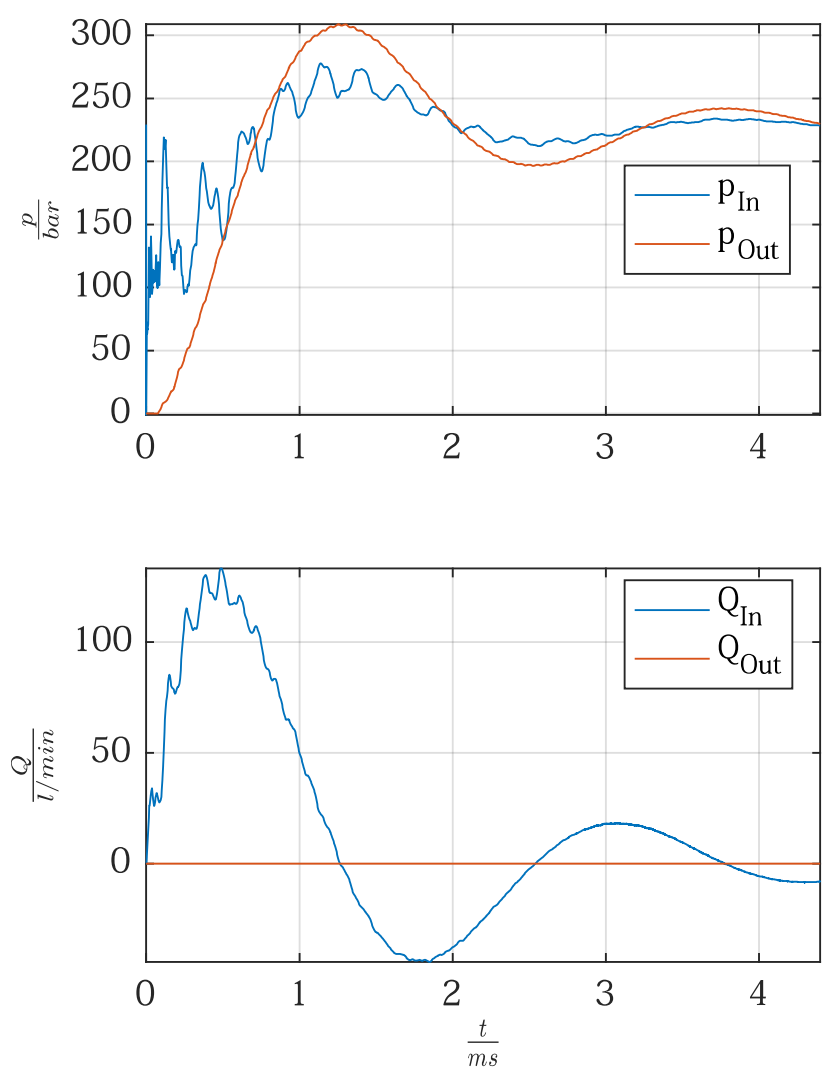

Figure 6: Simulation results: Valve Opening

A look on the flow rate chart in Fig. 6 reveals a maximum valve flow rate of approximately $120 \mathrm{l} / \mathrm{min}$ at around 0.5 milliseconds. The spatial pressure and flow rate distribution at this time is given in Fig. 7 where the sharp pressure drop at the orifice and the formation of a jet can be seen. 


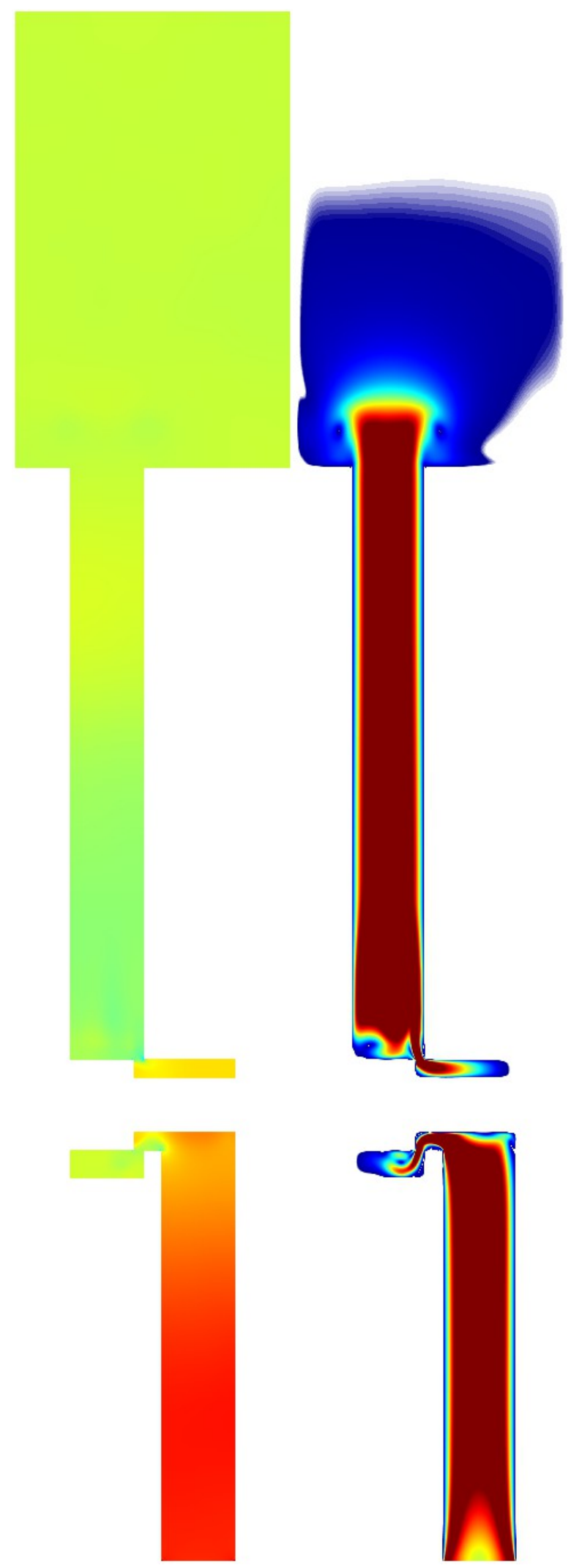

Figure 7: Pressure and velocity at the time of maximum valve flow rate around 0.5 milliseconds.

\section{Extraction of a frequency domain input- output model}

The information about pressures and flow rates at the subsystem input and output given in the time domain in Fig. 6 is now transferred to the frequency domain by Fast Fourier Transfrom (FFT). As the signals are neither periodic nor square integrable in time, the Fourier transform is not directly applicable. A simple trick is to differentiate both signals in time by just using the differences of successive values divided by the time step. These signals are transformed by FFT and the quotient of the frequency transformed output pressure divided by the frequency transformed input flow rate gives a transfer function describing the influence of the flow rate across the valve onto the effective piston face pressure and thus the piston force.

Figure 8 gives the resulting frequency domain information in the form of a Bode diagram commonly used in control theory. The blue line shows the frequency domain transfer function extracted from the Lattice-Boltzmann computation via Fast Fourier Transform, while the red line corresponds to a first order lumped parameter model treating the connection bore between valve and cylinder chamber and the chamber itself as one big control volume.
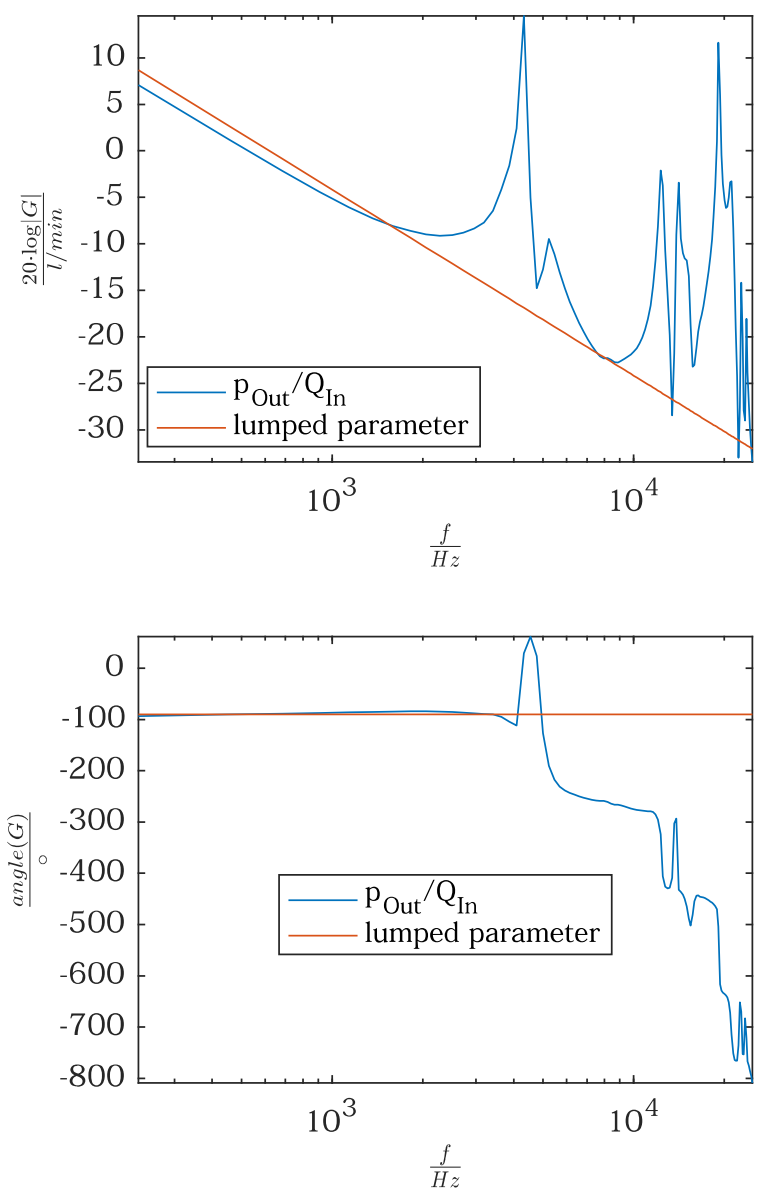

Figure 8: Simulation results: Valve Opening 
Obviously, the Lattice-Boltzmann results match very well with first order low frequency behavior. Around $4500 \mathrm{~Hz}$, the Lattice-Boltzmann results suggest the existence of a resonance due to wave propagation. This information could now be used to fit a low - say third order - model into the non-parametric frequency domain results presented in this paper.

In conclusion, the Lattice-Boltzmann approach can be shown to give feasible simulation times to calibrate a low order model, which can in turn be used for controller design or optimization purposes, where high dimensional computational fluid dynamics models are not usable under economical and time constraints.

\section{Conclusions and outlook}

In conclusion, the Lattice-Boltzmann approach can be shown to give very useful results to calibrate a low order model, which can in turn be used for controller design or optimization purposes, where high dimensional computational fluid dynamics models are not usable under economical and time constraints.

A major problem remains with respect to the stability limit preventing the application to fluid power systems in a realistic viscosity range. This issue is the topic of ongoing research and a follow-up publication with a more realistic example setting can be expected.

\section{References}

[1] J Hardy, Y Pomeau, and O de Pazzis. Time Evolution of a Two-Dimensional Classical Lattice System. Phys. Rev. Lett., 31(5):276-279, 1973

[2] J Hardy, O de Pazzis, and Y Pomeau. Molecular dynamics of a classical lattice gas: Transport properties and time correlation functions, Phys. Rev. A, 13(5):1949-1961, 1986

[3] U Frisch, B Hasslacher, and Y Pomeau. Lattice-Gas Automata for the Navier-Stokes Equation. Phys. Rev. Lett., 56(14):1505-1508, 1986

[4] G R McNamara, G Zanetti. Use of the Boltzmann equation to simulate lattice-gas automata. Phys. Rev. Lett., 61:2332-2335, 1986

[5] H E Merritt. Hydraulic Control Systems. John Wileys and Sons, Cincinnati, Ohio, 1967. ISBN 0-471-596175 .

[6] B Chopard, D Lagrava, J Latt, and O Malaspinas, A Lattice Boltzmann modeling of blood flow in cerebral aneurysms European Congress on computational methods in applied sciences and engineering (ECCOMAS), Lisbon" 2010

[7] FlowKit Sarl, Avenue de Chailly 23, 1012 Lausanne, Switzerland, PALABOS Software, Version 1.5r1, http://www.palabos.org/images/palabos releases/palab os-v1.5r1.tgz $\quad$ [accessed April $\left.15^{\text {th }}, \quad 2017\right]$ 